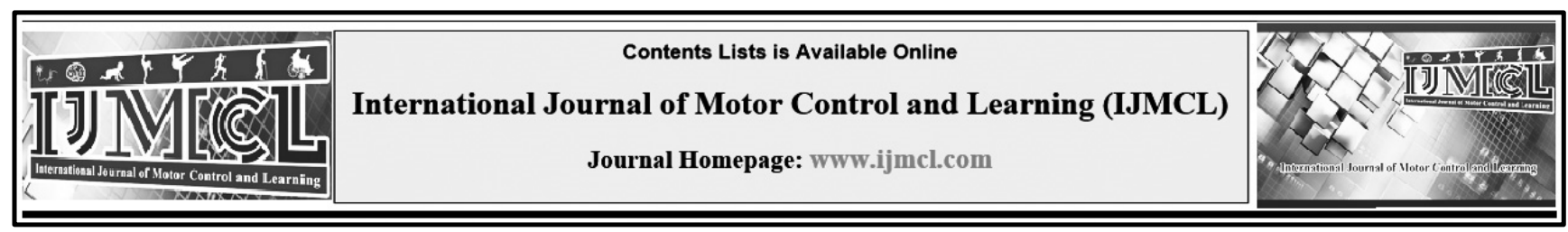

\title{
It is Time to Move from Motor Behavior and Sport Psychology Labs to Naturalistic Environments
}

\author{
Abbas Bahram ${ }^{a *}$

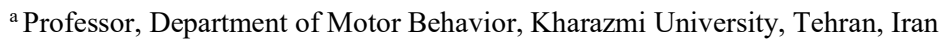 \\ * Corresponding E-mail: bahram $@$ khu.ac.ir \\ Department of Motor Behavior, Kharazmi University, Tehran, Iran.
}

Received: 2019/11/25

Accepted: 2020/02/09

Published: 2020/05/10

\section{DEAR EDITOR}

For many of those who are interested in the effects of focus of attention on skilled motor behavior, there is little or no doubt that adopting an external focus of attention (i.e., to movement effects), is a beneficial attentional strategy- compared with an internal focus (i.e., to body parts and body movements). This conclusion mainly comes from extensive experimental studies that have investigated the issue for about 24 years using different populations (e.g., typical and atypical individuals, children, young and older adults, males and females, athletes and non-athletes, novices and experts), and motor tasks (e.g., fine vs gross or open vs closed skills motor task) (Wulf, 2007, 2013, 2016). The constrained action hypothesis $(\mathrm{CAH})$ provides theoretical explanation for these findings as it theorizes that an external focus promotes movement automaticity while an IF disrupts automatic processing (Wulf, Shea, \& Park, 2001). This explanation has been supported in several laboratory studies measuring muscular activity (Kal, Van der Kamp, \& Houdijk,
2013; Lohse, Sherwood, \& Healy, 2010; Wulf, Dufek, Lozano, \& Pettigrew, 2010), workload (Raisbeck \& Diekfuss, 2017), and attentional demands (Wulf, McNevin, \& Shea, 2001).

While the findings and theoretical explanations regarding the superiority of an external focus of attention over an internal one look robust, these findings have been criticized for several reasons. For example, high reliance of studies on experimental designs (i.e., using laboratory-based studies and decontextualizing movement skills), extreme use of simple or very simplified motor tasks, and viewing dynamic and multidimensional phenomenon of attention as static and dichotomous are some of these limitations that may reduce the ecological validity of the findings dramatically (i.e., the extent to which the findings can transfer to naturalistic scenarios) (Collins, Carson, \& Toner, 2016; Toner, Montero, \& Moran, 2015; Toner \& Moran, 2015). For instance, the fact that experimental studies view attention as dichotomous (e.g., external, vs internal) may 
not represent what individuals (athletes) focus on or should focus on to support the highest level of performance. In other words, with using experimental works, other possible types of attentional strategies have largely been remained unknown (both of those attentional strategies that can promote or hinder skilled motor execution). In addition, in experimental studies, with adopting a static perspective, a single focus has been associated with a discrete action (e.g., "focus on your throwing arm while you are throwing") (Bernier, Trottier, Thienot, \& Fournier, 2016). This type of focus that is imposed by experimental conditions does not represent the natural conditions as does not consider either the preceding or succeeding foci, and also how these foci can affect skilled behavior. Therefore, a methodological shift from quantitative to qualitative research may be a better candidate to explore what focus of attentions is, and how athletes focus or should focus while they are performing their motor tasks in naturalistic environments.

Using qualitative techniques such as selfconfrontation interviews (Bahmani, Bahram, Diekfuss, \& Arsham, 2019)- that allows to capture real actions in natural, dynamic, and ever-changing conditions (and not artificial laboratory-based actions) - researchers have the opportunity to explore not only what athletes focus on in naturalistic environments, but also this methodology allows researchers to relate each single focus to each single movement phase (e.g., preparation phase, execution phase). Mixed method strategies that combine qualitative findings with quantitative measures, are very helpful as they allow researchers to gain more understanding regarding the phenomenon under study, by reducing the potential limitations of either quantitative, and qualitative works. With regards to this latest notion, we did a very recent mixed-method study on expert judo players (Bahmani et al., 2019). In the qualitative phase of the study, we aimed to understand how expert judo players direct their attention in different performance phases during natural competitions. As mentioned, in opposite to experimental designs, this qualitative portion of our study had several strengths including: 1) Allowing for perception-action coupling with investigating actions in real conditions. 2) Allowing to explore other possible types of foci 3 ) Considering the dynamic nature of focus. While our qualitative findings were very representative, this information were not sufficient to obtain a full picture of the story. We knew attentional strategies of expert judo players (i.e., what they attend to) during naturalistic conditions. We even knew the number of foci that were adopted in different performance phases. But what we did not know was that: How our judo players' attentional strategies (i.e., types, and number of foci) do affect their performance when they do their actions in naturalistic conditions? To provide answer to this questions, we added quantitative measures of performance effectiveness (i.e., scores obtained by performing each attack) and performance efficiency (participants' work-load in each attack) to our qualitative findings. Results indicated that reporting a lower quantity of attentional cues and higher percentage of focus on opponent resulted in higher performance effectiveness and less perceptions of workload. So, the study was novel as was the first one that was trying to relate natural attentional strategies to performance measures.

Research into sport offers a multitude of opportunities that are diverse in topic and broad in approach. A number of research strategies and associated research methods exist that allow for the exploration and explanation of sporting behavior from a range 
of perspectives. These could be observational if we were interested in coaching behavior, ethnographical if we wished to examine the socio-cultural functioning of a sports team, or experimental if our questions centered on performance enhancement. Each research strategy can be selected based on the nature of the inquiry and questions that need answering. As in all research, the selection of an appropriate research strategy from a clearly stated research question creates the link between aims and research methods (Smith, 2010). Several lines of investigation on attentional foci also remain to be addressed in future studies. First, few studies have tried to explore focus of attention in naturalistic environments (Bahmani et al., 2019; Bernier, Codron, Thienot, \& Fournier, 2011; Bernier et al., 2016; Nyberg, 2015), and thus much qualitative work is needed to understand the nature of attentional strategies of different sports and populations. Secondly, the study on expert judo players' indicated that focus on the opponent was a beneficial attentional strategy, however, this study failed to test the effect of other types of foci (e.g., internal focus) on skilled performance, mainly because the participants reported other foci very less frequently. In recent qualitative studies, it has been found that expert athletes in some sports like figure skating tend to focus internally to their body movements most frequently (Bernier et al., 2016; Nyberg, 2015). Based on these recent findings, it is possible that for these self-paced skills, and other sports with similar nature (e.g., gymnastic routines) adopting an internal focus of attention be a more beneficial attentional strategy. However, instead of merely relying on participants' reports, we need more rigorous methodological approaches such as what has been proposed by Bahmani et al (2019) to provide a linkage between participants' qualitative reports with quantitative indicators of performance success. Third, based on the sport under investigation, different types of foci may be explored by qualitative works. In this regard, while experimental studies have continuously investigated dichotomous foci (e.g., internal vs external; associated vs dissociative), we found five broad attentional categories that included several sub-categories and themes. This finding, along with previous studies (Bernier et al., 2016; Fairbrother, Post, \& Whalen, 2016), highlight that experts' focus of attention is not only more complex than what dichotomous laboratory-based studies typically investigate, but they are dynamic, flexible and shift depending on the phase of competition. So, it's time to move from motor behavior and sport psychology labs to naturalistic environments when studying the focus of attention.

\section{References}

1. Bahmani, M., Bahram, A., Diekfuss, J. A., \& Arsham, S. (2019). An expert's mind in action: Assessing attentional focus, workload and performance in a dynamic, naturalistic environment. Journal of sports sciences, 113.

2. Bernier, M., Codron, R., Thienot, E., \& Fournier, J. F. (2011). The attentional focus of expert golfers in training and competition: a naturalistic investigation. Journal of Applied Sport Psychology, 23(3), 326-341.

3. Bernier, M., Trottier, C., Thienot, E., \& Fournier, J. (2016). An investigation of attentional foci and their temporal patterns: A naturalistic study in expert figure skaters. The Sport Psychologist, 30(3), 256-266.

4. Collins, D., Carson, H. J., \& Toner, J. (2016). Letter to the editor concerning the article "Performance of gymnastics skill benefits from an external focus of attention" by Abdollahipour, Wulf, Psotta \& Nieto (2015). Journal of sports sciences, 34(13), 1288-1292.

5. Fairbrother, J. T., Post, P. G., \& Whalen, S. J. (2016). Self-Reported Responses to Player Profile Questions Show Consistency with the Use of Complex Attentional Strategies by Expert Horseshoe Pitchers. Frontiers in psychology, 7.

6. Kal, E., Van der Kamp, J., \& Houdijk, H. (2013). External attentional focus enhances movement automatization: A comprehensive test of the constrained action hypothesis. Human Movement Science, 32(4), 527-539.

7. Lohse, K. R., Sherwood, D. E., \& Healy, A. F. (2010). How changing the focus of attention affects 
performance, kinematics, and electromyography in dart throwing. Human Movement Science, 29(4), 542-555.

8. Nyberg, G. (2015). Developing a 'somatic velocimeter': the practical knowledge of free skiers. Qualitative Research in Sport, Exercise and Health, 7(4), 488-503.

9. Raisbeck, L. D., \& Diekfuss, J. A. (2017). Verbal Cues and Attentional Focus: A Simulated Target-Shooting Experiment. Journal of Motor Learning and Development, 5(1), 148-159.

10. Toner, J., Montero, B. G., \& Moran, A. (2015). Considering the role of cognitive control in expert performance. Phenomenology and the Cognitive Sciences, 14(4), 1127-1144.

11. Toner, J., \& Moran, A. (2015). Enhancing performance proficiency at the expert level: Considering the role of 'somaesthetic awareness'. Psychology of Sport and Exercise, 16, 110-117.

12. Wulf, G. (2007). Attention and motor skill learning: Human Kinetics.

13. Wulf, G. (2013). Attentional focus and motor learning: a review of 15 years. International Review of Sport and Exercise Psychology, 6(1), 77-104.

14. Wulf, G. (2016). An external focus of attention is a conditio sine qua non for athletes: a response to Carson, Collins, and Toner (2015). Journal of sports sciences, 34(13), 1293-1295.

15. Wulf, G., Dufek, J. S., Lozano, L., \& Pettigrew, C. (2010). Increased jump height and reduced EMG activity with an external focus. Human Movement Science, 29(3), 440-448.

16. Wulf, G., McNevin, N., \& Shea, C. H. (2001). The automaticity of complex motor skill learning as a function of attentional focus. The Quarterly Journal of Experimental Psychology Section A, 54(4), 1143-1154.

17. Wulf, G., Shea, C., \& Park, J.-H. (2001). Attention and motor performance: preferences for and advantages of an external focus. Research quarterly for exercise and sport, 72(4), 335-344. 